\title{
Extrinsic Modulation and Motor Pattern Generation in a Feeding Network: a Cellular Study
}

\author{
Volko A. Straub and Paul R. Benjamin \\ Sussex Centre for Neuroscience, University of Sussex, Falmer, Brighton, BN1 9QG, United Kingdom
}

Systems level studies have shown that the paired serotonergic cerebral giant cells (CGCs) of gastropod mollusks have important extrinsic modulatory actions on the central pattern generator (CPG) underlying rhythmic ingestion movements. Here we present the first study that investigates the modulatory actions of the CGCs and their released transmitter 5-HT on the CPG at the cellular level. In the snail, Lymnaea, motoneurons such as the B4, B8, and B4CL cells are part of the feeding CPG and receive serotonergic synaptic inputs from CGCs. These motoneurons were used to investigate the effect of serotonergic modulation on endogenous cellular properties of CPG neurons. Cells were isolated from the intact nervous system, and their properties were examined by pharmacological methods in cell culture. Motoneurons were also grown in coculture with CGCs to compare 5-HT effects with CGC stimulation. Three distinct modulatory effects of exogenously applied 5-HT/CGC activity were seen: all three motoneuron types were depolarized by

Investigations of neural circuits underlying motor behavior have been particularly successful in the case of central pattern generators (CPGs) (for review, see Orlovsky et al., 1999). One important principle that has emerged from the study of CPGs is that their activity is not fixed but liable to modification by central chemical modulation. Both the endogenous cellular properties of neurons may be affected and/or the strength of synaptic connections between component neurons of the CPG circuit. Central modulation can arise from outside the immediate CPG circuit ("extrinsic modulation") or results from the release of neuromodulatory substances from constituent neurons of the CPG circuit itself ("intrinsic modulation"; Katz and Frost, 1996).

Serotonin (5-HT) and other amines act as external modulators of CPGs in a wide variety of vertebrate and invertebrate animals (Harris-Warrick, 1988), but for a fuller understanding of this type of modulation there is a need for examples where the cellular actions of 5-HT can be linked to the function of serotonergic neurons in a behavioral circuit. The paired giant serotonergic neurons [metacerebral cells or cerebral giant cells (CGCs)] in the feeding systems of gastropod mollusks are an important example for this type of study because they provide the sole serotonergic input to the feeding $\mathrm{CPG}$, which simplifies the

Received Oct. 26, 2000; revised Dec. 14, 2000; accepted Dec. 19, 2000.

V.A.S. was supported by a grant from the Gottlieb Daimler and Karl Benz Foundation, and V.A.S. and P.R.B. were supported by a grant from the Biotechnology and Biological Sciences Research Council.

Correspondence should be addressed to V. A. Straub, Sussex Centre for Neuroscience, University of Sussex, Falmer, Brighton, BN1 9QG, UK. E-mail: V.Straub@sussex.ac.uk.

Copyright (C) 2001 Society for Neuroscience $0270-6474 / 01 / 211767-12 \$ 15.00 / 0$
5-HT for prolonged periods leading to firing. Conditional bursting accompanied this depolarization in the B4/B8 cells, but not in B4CL cells. The frequency of the bursting was increased with increased CGC tonic firing. An increase in the size of postinhibitory rebound (PIR) occurred with 5-HT application in all three cell types, because of an increase in a $\mathrm{CsCl}$-sensitive, hyperpolarization-activated inward current. Similar modulatory effects on membrane potential, endogenous bursting, and PIR properties could be observed in the intact nervous system and were necessary for motoneuron activation during feeding. Part of the systems gating and frequency control functions of the CGCs appear to be caused by these modulatory effects on feeding motoneurons.

Key words: Lymnaea; central pattern generator; neuromodulation; serotonin; conditional bursting; PIR; hyperpolarizationactivated inward current; cell culture

correlation between cellular activity/5-HT action and behavioral effects (Weiss and Kupfermann, 1976; Pentreath et al., 1982).

The role of CGC activity and 5-HT action in modulation has been most extensively investigated in the neuromuscular system controlling the feeding apparatus of the marine mollusk Aplysia (Weiss et al., 1978; Kupfermann and Weiss, 1981), but the CGCs in this animal and in another mollusk, Lymnaea, have additional important modulatory effects on motor pattern generation via their effects on the feeding CPG, including control of oscillator frequency and gating (Rosen et al., 1989; Yeoman et al., 1996). However, these central modulatory functions have so far only been analyzed at the circuit and behavioral level (McCrohan and Benjamin, 1980; Tuersley and McCrohan, 1989; Yeoman et al., 1996), without attempting to study 5-HT modulation of the intrinsic cellular properties of individual neurons.

The synaptic connectivity of the Lymnaea feeding CPG is well understood and is mainly composed of interneurons (for review, see Brierley et al., 1997b), but recently specific motoneuron types have been shown to play an important role in pattern generation via their electrotonic coupling to feeding CPG interneurons (Staras et al., 1998). These pattern-generating motoneurons are synaptic targets for the modulatory serotonergic CGCs in Lymnaea (McCrohan and Benjamin, 1980), and their large size makes them an ideal model system for our study. We show here that CGC activity and 5-HT application significantly modulates the intrinsic properties of retraction phase motoneurons necessary for their activation during feeding, causing prolonged depolarizations of membrane potential, triggering a conditional endogenous bursting property, and enhancing their postinhibitory rebound (PIR) properties. Similar types of effects were recorded in 
the intact ganglion, increasing significantly our understanding of the modulatory role of CGC activity and 5-HT at the circuit and behavioral levels.

\section{MATERIALS AND METHODS}

Experimental subjects and chemicals. Adult specimens of Lymnaea stagnalis were obtained from Blade Biological (Kent, UK). The animals were kept in large holding tanks containing copper-free water on a $12 \mathrm{hr}$ light/dark cycle and fed lettuce three times a week.

All chemicals were purchased from Sigma (Poole, UK) unless otherwise stated.

Dissection and intracellular recording techniques. All dissections were performed in HEPES-buffered saline containing (in $\mathrm{mM}$ ): $\mathrm{NaCl}$ (50), $\mathrm{KCl}$ (1.6), $\mathrm{MgCl}_{2}$ (2), $\mathrm{CaCl}_{2}$ (3.5), and HEPES (10), pH 7.9, in distilled water. The CNS, consisting of the circumesophageal ganglionic ring (cerebral, pedal, pleural, parietal, and visceral ganglia) and the buccal ganglia together with a short stretch of esophagus, was isolated from the snail. The preparation was pinned down in a Sylgard-coated dish filled with HEPES-buffered saline with the dorsal surface facing up.

Standard intracellular recording techniques were used to record simultaneously from up to four individual neurons. The recording electrodes were pulled from $2 \mathrm{~mm}$ glass capillaries with inner filament (GC200F-15; Clarks Electromedical, Reading, UK) and filled with $4 \mathrm{M}$ potassium acetate (electrode resistance, $30-50 \mathrm{M} \Omega$ ). Signals were fed into standard intracellular recording amplifiers, digitized with a CED 1401plus interface (Cambridge Electronics Design, Cambridge, UK), and visualized and stored on a personal computer (PC) using Spike2 software (Cambridge Electronics Design). Penetration of neurons was facilitated by incubation of the isolated nervous system in a protease solution (Sigma type XIV; $1 \mathrm{mg} / \mathrm{ml}$ in HEPES-buffered saline) for $5 \mathrm{~min}$. The protease treatment was stopped by extensively washing the preparation with HEPES-buffered saline.

The effects of bath-applied 5-HT (0.1 mM, flow rate $3-4 \mathrm{ml} / \mathrm{min})$ on retraction phase motoneurons in isolated buccal ganglia were studied after the cerebral-buccal connectives were cut and the circumesophageal ganglionic ring was removed from the recording chamber. Furthermore, the buccal ganglia were superfused constantly with tubocurarine $(0.1$ $\mathrm{mM}$; d-TC) to eliminate spontaneous and 5-HT-induced fictive feeding activity by blocking cholinergic synaptic outputs from protraction phase interneurons such as N1Ms and slow oscillator (SO) (Elliott and Kemenes, 1992; Yeoman et al., 1996).

Identification and electrophysiological properties of neurons in the intact nervous system. Extensive work on the feeding system of the pond snail Lymnaea stagnalis has led to the identification of many of its components including motoneurons, CPG interneurons and modulatory neurons, and the characterization of their connectivity (Benjamin and Elliott, 1989; Brierley et al., 1997b). Most of the larger motoneurons, among those the retraction phase motoneurons $\mathrm{B} 4, \mathrm{~B} 8$, and $\mathrm{B} 4 \mathrm{CL}$ used in this study, can be identified visually after removal of the outer connective tissue. For example, B4, B8, and B4CL neurons are found in characteristic positions in the medioposterior quadrant of the buccal ganglia (Fig. $1 A$ ). These neurons form an extensive network of electrotonically coupled neurons (Fig. 1C; Benjamin and Rose, 1979). There are up to six B4CL neurons in each buccal ganglion that fire together during the rasp phase of the feeding cycle. The B4 and B8 neurons are pairs of (left and right) individually identifiable swallow phase motoneurons, that are strongly electrotonically coupled to each other and have similar electrical properties (Benjamin and Rose, 1979). The B4/B8 and B4CL motoneurons all receive consecutive phases of inhibitory synaptic inputs from the $\mathrm{N} 1$ and N2 CPG interneurons before they become activated when the buccal mass is retracted, either late in the rasp phase (B4CL) or at the start of the swallow phase (B4, B8) of the feeding cycle (Fig. 1B).

The feeding CPG interneurons can be divided into three main types, $\mathrm{N} 1, \mathrm{~N} 2$, and $\mathrm{N} 3$. The $\mathrm{N} 1$ neurons are active during the protraction phase, $\mathrm{N} 2$ neurons during the rasp phase, and N3 neurons during the swallow phase (Rose and Benjamin, 1979, 1981a,b). Each of the three main classes consists of at least two subtypes, medial N1 (N1M) and lateral N1 (N1L) (Yeoman et al., 1995), dorsal N2 (N2d) and ventral N2 (N2v) (Brierley et al., 1997a), phasic N3 (N3p) and tonic N3 (N3t) (Elliott and Benjamin, 1985). Both, N1 and N2 type feeding interneurons form monosynaptic inhibitory connections with retraction phase motoneurons, whereas N3 type interneurons provide an inhibitory chemical synaptic input and are electrotonically coupled to the B4/B8, B4CL retraction phase motoneurons (Fig. 1C; Staras et al., 1998). It is via these electro-
A
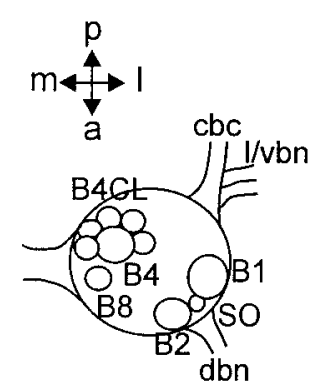

B SO-driven fictive feeding

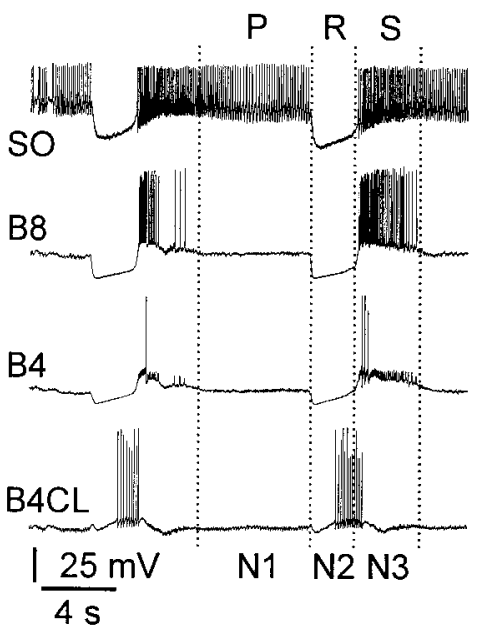

C

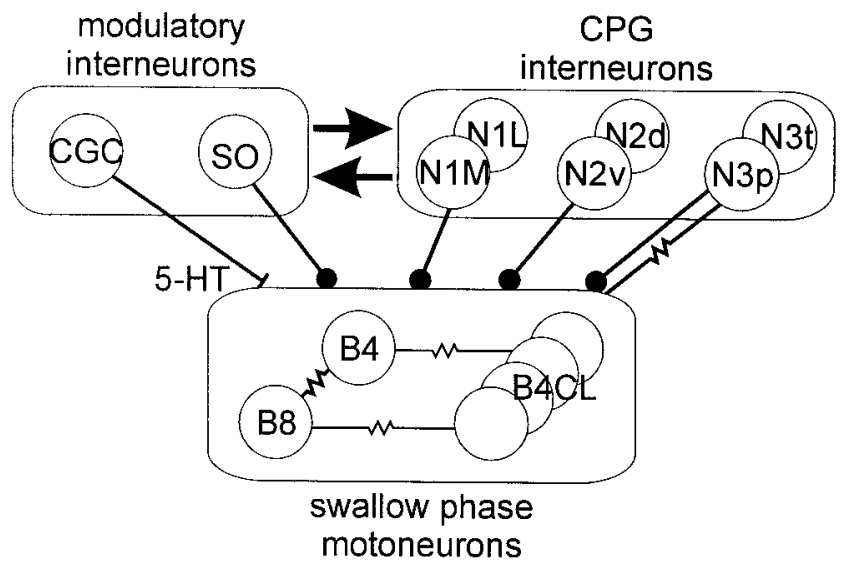

Figure 1. Location, firing patterns, and synaptic connectivity of retraction phase neurons in the buccal ganglia of Lymnaea. A, Schematic dorsal view of the left buccal ganglion showing the soma positions of $\mathrm{B} 4, \mathrm{~B} 8$, and $\mathrm{B} 4 \mathrm{CL}$ retraction phase neurons. The position of the $\mathrm{B} 1$ and $\mathrm{B} 2$ neurons is shown for reference purposes. The slow oscillator $(S O)$ can be located either in the left or right buccal ganglion between the B1 and B2 neuron. $c b c$, Cerebrobuccal connective; $l / v b n$, lateral/ventral buccal nerve; $d b n$, dorsal buccal nerve; $a$, anterior; $p$, posterior; $l$, lateral; $m$, medial. $B$, Simultaneous recording from retraction phase motoneurons B4CL, B4, and B8 in the isolated nervous system showing two cycles of fictive feeding. The fictive feeding pattern was driven by injection of a constant depolarizing current into the SO (top trace). The three phases of the feeding pattern ( $\mathrm{P} / \mathrm{N} 1$ protraction, $\mathrm{R} / \mathrm{N} 2$ rasp, and $\mathrm{S} / \mathrm{N} 3$ swallow) are marked above and below the traces. $C$, Schematic diagram of the known connections between retraction phase motoneurons, feeding CPG interneurons, and modulatory interneurons. Circles indicate inhibitory synapses, bars indicate excitatory synapses, and resistor symbols indicate electrotonic coupling.

tonic connections that the retraction phase motoneurons play a role in motor pattern generation with the B4/B8 and B4CL motoneurons contributing significantly to the maintenance and phase setting of the N3 phase CPG interneurons.

The serotonergic CGCs are a symmetrical pair of individually identifiable giant neurons located in the anterior lobe of the cerebral ganglia (McCrohan and Benjamin, 1980). Left and right cells usually fire synchronously in a continuous slow single-spiking pattern and this enables them to be identified with certainty in the isolated brain preparation. The CGCs have weak, mainly excitatory, chemical synaptic connections with motoneurons (McCrohan and Benjamin, 1980) and CPG interneurons (Yeoman et al., 1996). The synaptic responses can be mimicked by application of 5-HT and blocked by 5-HT receptor antagonists (Tuersley 
and McCrohan, 1989; Yeoman et al., 1994a). Injection of 5,6dihydroxytryptamine into intact snails reduced 5-HT levels in the CGCs, and this reduced the proportion of snails responding to natural food stimuli and/or the frequency of ingestion (Kemenes et al., 1990). Fine wire recording from the CGCs in the intact snail combined with systems level pharmacological analysis (blocking of 5-HT receptors) suggested that the CGCs have a gating/permissive function in the feeding system. A minimum level of tonic CGC firing $(\sim 7$ spikes/min) was required to support the food-driven consummatory phase of feeding in the intact snail or in an SO-driven fictive feeding rhythm in the isolated preparation (Yeoman et al., 1994a). An additional modulatory role in controlling the frequency of the feeding pattern occurred in the 7-40 spikes/min CGC firing range (Yeoman et al., 1994a,b). Although it is known that blocking 5-HT receptors in the feeding network prevented the normal gating and frequency actions of the CGCs, it is not known how these functions are implemented at the cellular level.

Isolation and culture of identified neurons. The cell culture procedure was modified from the protocol of Ridgway et al. (1991). Media used included normal saline (NS), antibiotic saline (ABS), defined medium (DM), and conditioned medium (CM). Normal saline used in cell culture experiments contained the same salt concentrations as the snail HEPESbuffered saline described above, but was made up in culture grade water, whereas ABS also contained gentamycin $(150 \mu \mathrm{g} / \mathrm{ml})$. DM was prepared by mixing $100 \mathrm{ml}$ of special L-15 medium (Life Technologies, Paisley, UK), $80 \mathrm{ml}$ of NS, and $120 \mathrm{ml}$ of culture grade water and by adding glutamine $(30 \mathrm{mg})$, glucose $(16.2 \mathrm{mg})$, and gentamycin $(600 \mu \mathrm{l}$ of 10 $\mathrm{mg} / \mathrm{ml}$ stock) to the solution. For CM preparation, isolated brains that had been washed extensively in ABS were incubated in DM (two brains per milliliter). After $3 \mathrm{~d}$ of incubation, the $\mathrm{CM}$ was sterile-filtered (Millex-GV, $0.22 \mu \mathrm{m}$; Millipore, Bedford, MA). Aliquots of CM (1 ml) were pipetted directly onto culture dishes (Falcon 3001; Becton Dickinson, Rutherford, NJ) coated with poly-L-lysine (15-30 kDa; $1 \mathrm{mg} / \mathrm{ml}$ in 15 $\mathrm{mM}$ Tris buffer), and equal amounts of DM were added. The culture dishes were stored at $-20^{\circ} \mathrm{C}$ and thawed $2-3 \mathrm{hr}$ before use.

The isolation of individual neurons was performed in a laminar flow cabinet after the isolated nervous system had received one of two alternative enzymatic treatments designed to soften the inner connective tissue. The first type of enzyme treatment consisted of incubation in trypsin $(0.67 \mathrm{mg} / \mathrm{ml})$ and collagenase/dispase $(1.33 \mathrm{mg} / \mathrm{ml}$; Boehringer Mannheim, Indianapolis, IN) in DM for $30 \mathrm{~min}$ at room temperature. This treatment was followed by $10 \mathrm{~min}$ incubation in soybean trypsin inhibitor $(1 \mathrm{mg} / \mathrm{ml}$ in $\mathrm{DM})$.

For the second type of enzyme treatment, the isolated nervous system was incubated for $45 \mathrm{~min}$ in a protease solution (Sigma type VIII; 1 $\mathrm{mg} / \mathrm{ml}$ in NS) and then washed in ABS. In our experience, this treatment facilitated the isolation of individual neurons with very long lengths of primary neurites.

After either of the two enzyme treatments, the isolated nervous system was pinned out in a dissection dish filled with high-osmolarity DM (30 $\mathrm{mm}$ glucose in DM). CGC, B4, B8, and B4CL neurons were visually identified according to their size and characteristic position (see Identification and electrophysiological properties of neurons in the intact nervous system). Their cell bodies were exposed by mechanically disrupting the inner connective tissue and then removed, together with their main processes, by gentle suction with a fire-polished micropipette (tip diameter, 100-200 $\mu \mathrm{m}$ ) prepared from $1.5 \mathrm{~mm}$ glass tubing (GC150T-10; Clark Electromedical Instruments) that had been coated with Sigmacote. After isolation, neurons were transferred onto culture dishes and cultured at $20^{\circ} \mathrm{C}$ from 1 to $5 \mathrm{~d}$.

Electrophysiological and pharmacological studies on cultured neurons. For intracellular recordings from isolated neurons, culture dishes containing either individual neurons or networks of isolated neurons were placed on the stage of an inverted microscope (Nikon Diaphot) that was equipped with a custom-built, gravity-fed perfusion system. The culture dishes were perfused with NS at a flow rate of 1-2 $\mathrm{ml} / \mathrm{min}$ throughout the experiment. Cell bodies were impaled with microelectrodes pulled from $1 \mathrm{~mm}$ capillaries (GC100F-10; Clark Electromedical Instruments) and filled with saturated potassium sulfate (tip resistance, 20-30 M $\Omega$ ). The electrodes were manipulated with three hydraulic manipulators (MW-3 and MO-300; Narishige, Tokyo, Japan) mounted to the stage of the microscope that enabled the independent control of up to three recording and/or perfusion pipettes. The intracellular signals were amplified using Axoclamp2-B (Axon Instruments, Foster City, CA) and Neurolog NL102 (Digitimer; Welwyn Garden City, UK) amplifiers, output to a storage oscilloscope (5115 Tektronix), and stored on a DAT recorder (Biological
DTR-1801; Biological Science Instruments, Claix, France). Amplified signals were also digitized using a DigiData 1200 interface (Axon Instruments) and stored on a PC. Twin-electrode voltage clamp (TEVC) recordings of single neurons were conducted using the Axoclamp2-B amplifier controlled by pClamp6 software (Axon Instruments) via the DigiData 1200 interface.

The effects of 5-HT $(0.1 \mathrm{~mm})$ were tested by focal application from a micropipette ( $1 \mathrm{sec}$ pulses at 6-12 psi) using a Picospritzer (General Valve, Fairfield, NJ) or by perfusing the recording chamber with 5-HT solutions in NS $(0.1-100 \mu \mathrm{M})$.

Statistical analysis of the data were performed using the SPSS software package (version 8.0 for Windows; SPSS, Chicago, IL). Paired $t$ tests and one-way ANOVA procedures followed by post hoc Tukey tests for multiple comparison were used when appropriate.

\section{RESULTS \\ 5-HT induces bursting in isolated retraction phase motoneurons}

Three types of retractor motoneurons (B4CL, rasp phase; B4/B8, swallow phase) contribute to motor pattern generation (Staras et al., 1998). We used these neurons as a model system to study the effect of serotonergic modulation on their cellular properties, because previous studies at the systems level have shown that 5-HT has important modulatory effects on the feeding CPG. For this purpose, identified neurons were isolated from the intact nervous system and cultured individually or in pairs. All results on isolated neurons presented in this study were obtained from neurons that were cultured for periods between 1 and $5 \mathrm{~d}$. Already after $1 \mathrm{~d}$ the neurons had regenerated extensive processes. We found no evidence for any consistent changes in the cellular properties of the isolated neurons during the $5 \mathrm{~d}$ period in culture.

Individually cultured B4, B8, and B4CL cells usually displayed a transient period of spontaneous activity after microelectrode impalement before becoming quiescent and settling at a stable membrane potential. During the transition from activity to eventual silence, approximately one-fifth of all cultured B4 and B8 swallow phase motoneurons (33 of 151 and 8 of 37, respectively) showed patterns of regular bursting activity (Fig. $2 A$ ). In contrast, B4CL neurons $(n=84)$ never displayed regular bursting after impalement but showed only a gradual decrease in firing frequency until spontaneous activity ceased completely (Fig. $2 A$ ). In some cases, B4CL neurons generated spike doublets or irregular changes in firing frequency during this period of activity (data not shown). However, these events were not superimposed on rhythmic oscillations of the membrane potential that were observed in bursting B4 and B8 neurons. This observation indicated that B4 and B8 neurons, but not B4CL neurons, possess some tendency toward endogenous bursting.

Sustained bursting was induced more reliably in single B4 and B8 neurons by bath application of 5-HT $(0.1 \mathrm{~mm}, 60 \mathrm{sec})$. The sustained bursting activity was superimposed on a prolonged depolarization (Fig. 2B). Both the 5-HT-induced depolarization and bursting activity could last for periods in excess of $10 \mathrm{~min}$, considerably outlasting the immediate period of application even though the culture dish was continuously washed with normal saline. All the B4 $(n=7)$ and B8 cells $(n=8)$ tested showed some rhythmic oscillation of the membrane potential in response to 5-HT. These endogenous bursting properties appeared to be conditional on the presence of 5-HT, because they could not be induced in isolated B4 and B8 neurons by steady current injection ( $n>10$ for both cell types; data not shown).

There was considerable variation between individual cells in the duration of the bursts, with B4 or B8 bursts varying from a 
Figure 2. Activity patterns of isolated retraction phase motoneurons in cell culture. $A$, Activity of isolated B4, B8, and B4CL neurons after impalement with a microelectrode. Arrowheads above the records indicate the time of impalement. Note the transient periods of bursting activity in the $\mathrm{B} 4$ and $\mathrm{B} 8$, but not the B4CL neuron. $B$, Bath application of 5-HT $(0.1 \mathrm{~mm}, 60 \mathrm{sec})$ caused prolonged depolarizations in isolated B4, B8, and B4CL neurons that induced spiking activity. After an initial period of tonic activity, 5-HT induced bursting activity in the B4 and B8 neurons, but not the B4CL neuron, that lasted for $>10 \mathrm{~min}$. $C$, Simultaneous records from a pair of cocultured B4 and B8 neurons that had formed a strong electrotonic connection. Bath application of 5-HT $(0.1 \mu \mathrm{M}$ for $4 \mathrm{~min})$ caused a prolonged depolarization of both neurons that triggered spiking activity first in the $\mathrm{B} 8$ and then the B4 neuron. After an initial period of tonic activity, synchronized bursting occurred in both neurons that lasted for $>20 \mathrm{~min}$ after the start of the 5-HT application. The three short records on the right illustrate the electrotonic coupling between the two neurons and its ability to synchronize activity in the two cells. Injection of a hyperpolarizing current into the B4 neuron caused a similar, but smaller hyperpolarization in the B8 neuron. Conversely, induced bursts of activity in either of the two neurons evoked simultaneous bursts in the other neuron. $D$, Simultaneous records from a pair of cocultured $\mathrm{B} 4$ and $\mathrm{B} 4 \mathrm{CL}$ neurons that had formed an electrotonic connection. Bath application of 5-HT (1 $\mu \mathrm{M}$ for $45 \mathrm{sec})$ caused a prolonged depolarization in both neurons that triggered spiking activity in both neurons. The B4 neuron, but not the B4CL neuron (despite its electrotonic connection to the B4 neuron) displayed a period of bursting activity during the 5-HT-induced depolarization. Negative current pulses injected into either of the two neurons evoked similar, but weaker responses in the other neuron illustrating the electrotonic coupling between the two neurons.
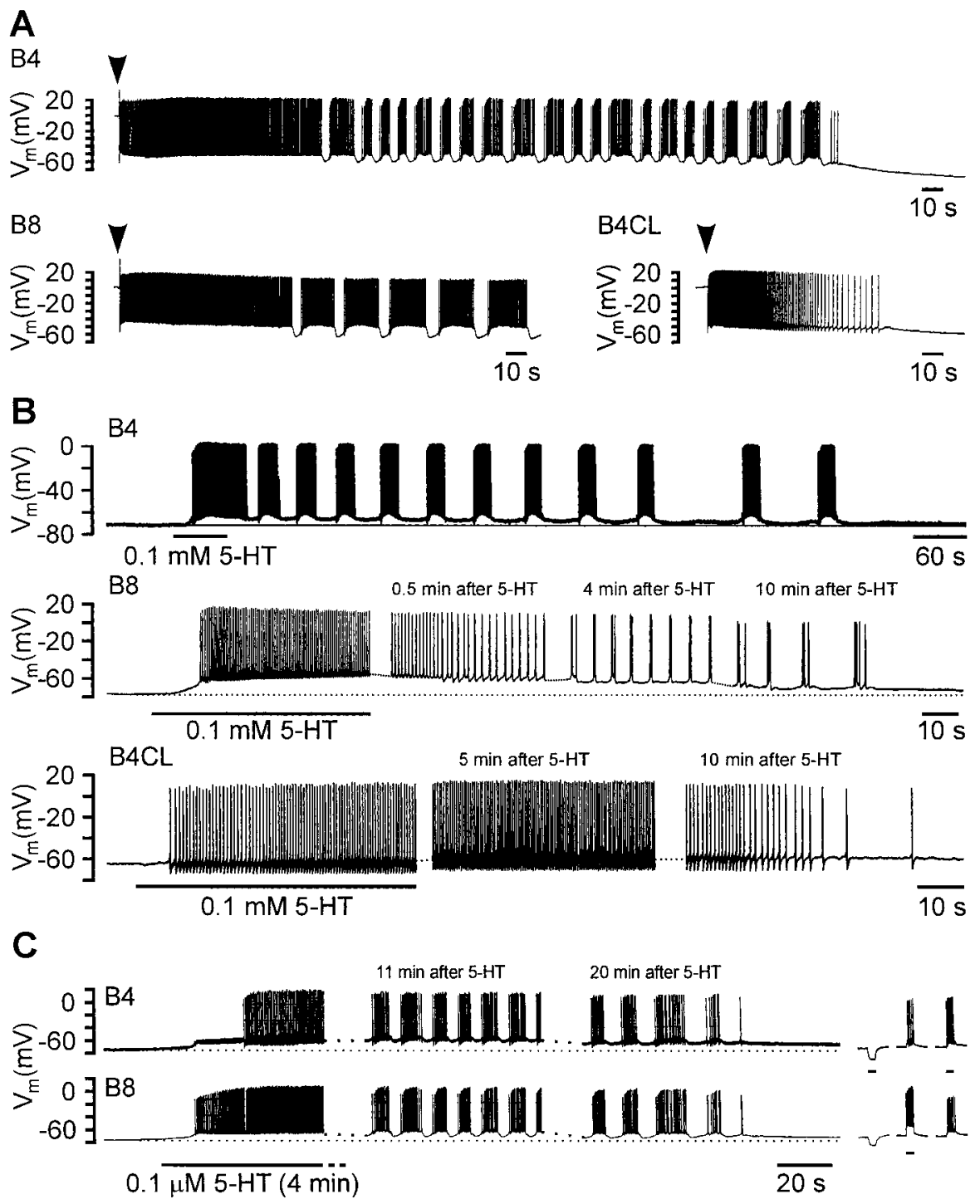

D

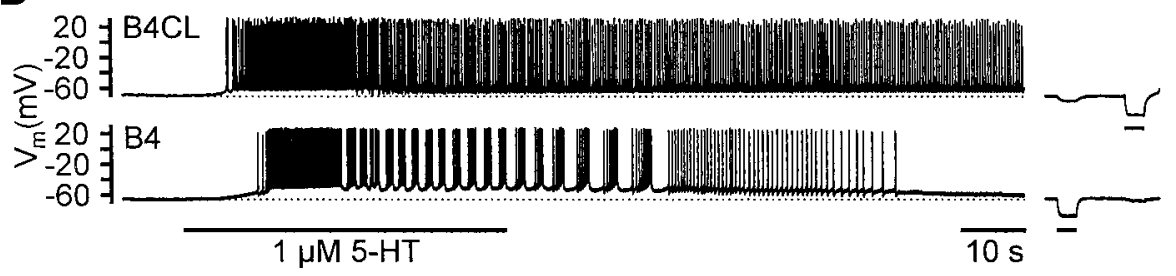

minimum of $\sim 1 \mathrm{sec}$ (e.g., Fig. $2 B, B 8$ ) to $>20 \mathrm{sec}$ (e.g., Fig. $2 B$, B4). Lower 5-HT concentrations $(0.1-10 \mu \mathrm{M})$ could also induce sustained bursting (Fig. 2C,D), but were less reliable. When 5-HT was bath-applied for up to 4 min to a cocultured pair of B4 and B8 neurons, both cells were induced to burst with activity showing close synchrony (Fig. 2C). The synchronization was probably caused by the strong electrotonic connection that formed between them in cell culture as indicated by the records showing that artificially depolarizing one cell could induce a burst of spikes in the other (Fig. 2C, right). The electrotonic coupling found in $75 \%$ of B4-B8 pairs in culture $(n=8)$ recapitulates that recorded between B4 and B8 in the intact ganglion (Benjamin and Rose, 1979).

Bath application of 5-HT ( $0.1 \mathrm{~mm}$ or $1 \mu \mathrm{M}, 30-60 \mathrm{sec})$ to the
B4CL cell type again elicited depolarizing responses (10 of 13 tested). This induced prolonged tonic spike activity with the occasional occurrence of spike doublets, but unlike the B4/B8 cells, never resulted in regular bursting (Fig. $2 B$ ). In the example shown in Figure $2 D$, the B4CL neuron was cocultured with a bursting B4 neuron, which had reformed an electrotonic connection with the B4CL neuron similar to those found in the intact nervous system (Benjamin and Rose, 1979). Here, even in the presence of a bursting B4 neuron, 5-HT failed to induce bursting in the B4CL neuron.

A shorter $1 \mathrm{sec}$ focal application of 5-HT (0.1 mM) could also induce bursting in single isolated B4 (Fig. 3 $\mathrm{Ai}$ ) and B8 neurons (data not shown), consistent with bath application. Again, this never occurred in B4CL neurons (Fig. 3Aii; $n=25$ ). The propor- 

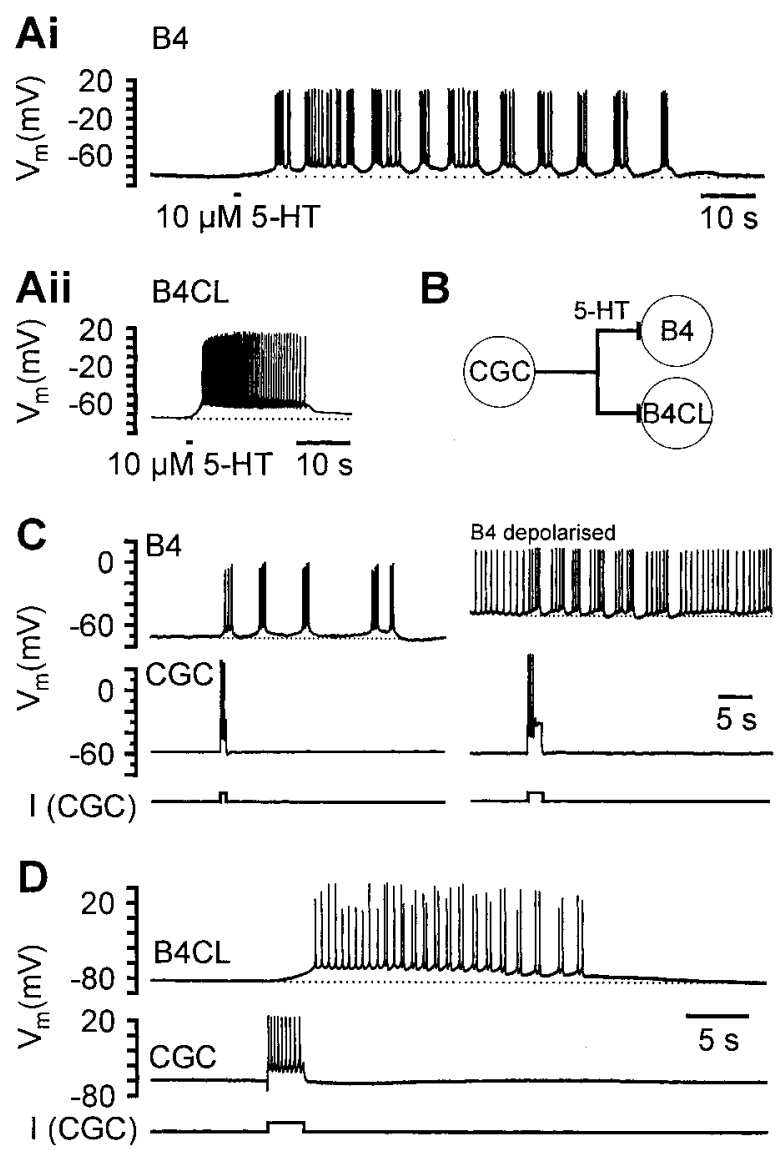

Figure 3. Responses of isolated B4 and B4CL neurons to 5-HT pulses and bursts of CGC activity. Ai, A brief focal application of 5-HT (10 $\mu \mathrm{M}$, $1 \mathrm{sec}$ ) caused a prolonged depolarization and bursting activity in an isolated B4 neuron that lasted for $>50 \mathrm{sec}$. Aii, An identical 5-HT application to an isolated B4CL neuron evoked a strong, but shorter depolarization that triggered a single burst of activity lasting for $20 \mathrm{sec} . B$, Schematic diagram showing the monosynaptic excitatory chemical synapses from CGC to B4 and B4CL neurons. $C$, Simultaneous records from a pair of cocultured CGC and B4 neurons that had reformed a chemical synapse in cell culture. Left panel, Injection of a brief positive current pulse into the CGC neuron directly triggered a burst of three CGC spikes. The burst of CGC activity also elicited a longer-lasting depolarization in the B4 neuron that caused four bursts of activity over a period of $30 \mathrm{sec}$. Right panel, The B4 neuron was constantly depolarized by injection of a weak positive current that induced tonic firing. A brief burst of three CGC action potentials temporarily switched the B4 activity pattern from tonic to bursting before it reverted back to tonic activity after $\sim 25 \mathrm{sec} . D$, Simultaneous records from a pair of cocultured CGC and B4CL neurons that had reformed a chemical synapse in cell culture. A burst of CGC spikes induced by a positive current pulse caused a substantial depolarization of the B4CL neuron that triggered B4CL activity lasting for $22 \mathrm{sec}$. The B4CL neuron generated spike doublets during this period of activity but showed no pronounced bursting as observed in B4 neurons.

tion of B4 and B8 cells that responded to focally applied 5-HT by bursting was much lower than with bath application. For instance, 5 -HT-induced bursting only occurred in $21 \%$ of B4 neurons tested (7 of 33).

\section{Bursting in B4 is triggered by CGC activity}

In the intact nervous system CGC neurons are the sole source of serotonergic inputs to the retraction phase motoneurons and the feeding CPG in general (Kemenes et al., 1989). Their ability to mimic the effects of 5-HT was tested in experiments in which they were cocultured with B4/B4CL neurons. Chemical synapses were formed in $\sim 50 \%$ of CGC-B4 pairs $(n=35)$ and $\sim 30 \%$ of CGC-B4CL pairs $(n=14)$.

Bursts of current-evoked spikes in the CGCs could induce sequences of B4 bursting in silent B4 neurons or could change the activity of tonically active B4 neurons into bursting. This is illustrated by the example shown in Figure $3 C$ (left panel) in which a brief burst of three CGC action potentials triggered a series of five bursts of B4 action potentials over a period of $\sim 30$ sec, with depolarization above the resting level maintained for the same period of time. Tonic single spiking induced by injection of a constant small positive current in the same B4 neuron could be switched temporarily to bursting by a brief burst of CGC action potentials (Fig. 3C, right panel). The observation that simple depolarization of the $\mathrm{B} 4$ membrane potential induces only tonic activity, whereas CGC activity can elicit bursting activity in the quiescent and tonically active B4 neuron, supports our hypothesis that B4 bursting is conditional and depends on the presence of CGC activity and 5-HT.

In B4CL neurons, CGC activity could also elicit a prolonged depolarization and trigger activity in the B4CL neurons (Fig. $3 D$ ). This could be in the form of spike doublets, but the B4CL neurons never showed prolonged bursts of activity superimposed on pronounced membrane potential oscillations observed in the B4 neurons.

During feeding movements in the intact snail, the CGC neurons fire tonically at an average rate of between 7 and 20 spikes/ min, although occasionally higher rates of up to 40 spikes/min have been recorded (Yeoman et al., 1994a). Related studies in the isolated nervous system have shown that $>7$ spikes/min the CGCs exert a frequency control function on the fictive feeding rate (Yeoman et al., 1996). Here we show that the rate of CGC activity can directly control the frequency of B4 bursting in culture as well. For this purpose, activity in isolated CGCs was set at various tonic levels within the physiological range by current pulse injection, and the effects on the bursting of cocultured B4 cells were systematically examined $(n=3)$. In the example shown in Figure 4 the CGC effect on B4 bursting was tested at CGC firing rates from zero up to $60 \mathrm{spikes/min}$ for standard periods of 250 sec. Intervals in excess of 8 min were allowed between successive episodes of CGC stimulation, during which bursting in the B4 neuron returned to control levels.

In the absence of CGC spike activity, the cocultured B4 neuron still generated periodic burst activity (control in Fig. $4 A$ ) caused by the residual effects of previous CGC activity (e.g., spontaneous CGC activity after impalement, previous CGC stimulation to test CGC-B4 synapse). Increasing CGC activity progressively increased the frequency and strength of the spontaneous B4 bursts (Fig. $4 A$ ). The results were analyzed quantitatively by measuring the frequency of B4 bursts, the interburst interval between B4 bursts, and the frequency of B4 spiking within the four B4 bursts immediately before CGC stimulation (control) and comparing their mean values with the same parameters measured for the last four bursts of B4 activity during CGC stimulation (test). The results for this cell are presented as difference scores (test minus control) in Figure $4 B-D$. Paired $t$ test comparisons of the control versus the test values were performed for statistical analysis. CGC stimulation at a rate of 12 spikes/min increased significantly the B4 burst frequency by $0.5 \pm 0.14$ bursts/min on average $(t$ test, $p=$ 0.04; Fig. $4 B$ ). Stronger increases in the rate of CGC firing to 24 spikes/min and 60 spikes/min had more substantial effects on B4 bursting, increasing the mean burst frequency by $1.0 \pm 0.17$ bursts $/ \min (t$ test, $p=0.01)$ and $1.6 \pm 0.23$ bursts $/$ min $(t$ test, $p=$ 
Figure 4. Effects of CGC firing rates on B4 bursting in cocultured CGC and B4 neurons that had reformed a chemical synapse in cell culture. $A$, The top record shows the spontaneous activity pattern of the $\mathrm{B} 4$ neuron in the absence of CGC activity (CGC recording not shown). The subsequent three pairs of records illustrate the changes in the activity pattern of the same B4 neuron, when the CGC neuron was stimulated at various frequencies $(12,24$, and 60 pulses/min) by repetitive short superthreshold depolarizing current pulses $(200 \mathrm{msec})$ that triggered individual CGC spikes. $B-D$, Quantitative analysis of the changes in $\mathrm{B} 4$ bursting induced by CGC activity at various frequencies shown in $A$. The changes in frequency of B4 bursts $(B)$, B4 interburst intervals $(C)$, and $\mathrm{B} 4$ spike frequency during bursts were determined for the last four B4 bursts during CGC stimulation compared with the four B4 bursts before the start of each CGC stimulation (see Results for further interpretation and details of statistical analysis, ${ }^{*} p<0.05 ;{ }^{* *} p<0.01$; $* * * p<0.001)$.
A
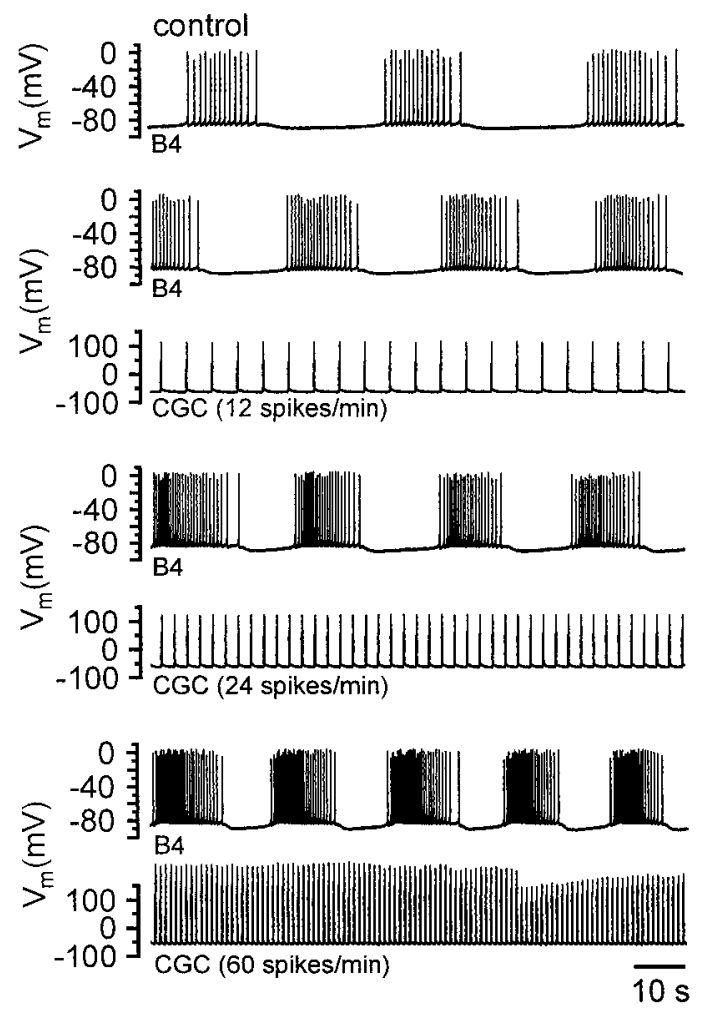

B

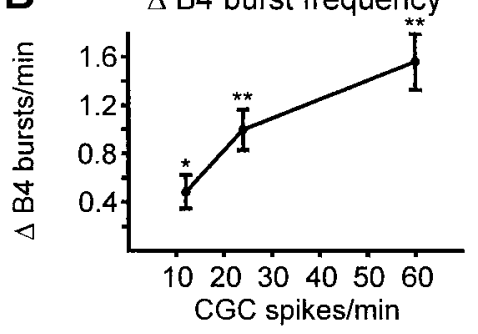

C $\Delta \mathrm{B} 4$ inter-burst interval CGC spikes/min
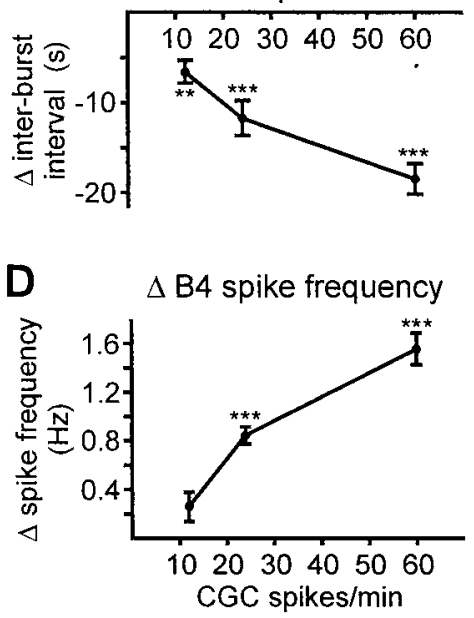

0.01), respectively. The increases in B4 burst frequency resulted mainly from a significant decrease in length of the interburst intervals ( $t$ tests, $p \leq 0.01$ for all three CGC firing rates; Fig. 4C). Furthermore, it was also noted that the frequency of B4 spiking within the bursts were increased significantly when CGC activity was raised to 24 or 60 spikes/min, but not at 12 spikes/min (12 CGC spikes/min, $t$ test, $p=0.12 ; 24$ and 60 CGC spikes $/ \mathrm{min}, t$ test, $p=0.001$; Fig. 4D).

Further analysis using ANOVA tests revealed significant differences between the effects that the three levels of CGC activity $(12,24$, and 60 spikes/min) had on the B4 burst frequency, the B4 interburst interval, and the B4 spike frequency within a burst (ANOVA: $F_{(2,9)}$ values from 8.8 to $34.8, p$ values $<0.01$ for all three B4 parameters measured). Post hoc multiple comparisons using a Tukey honest significant difference (HSD) test showed that the differences in the B4 spike frequency changes within a burst were significant at the $5 \%$ level between all three levels of CGC activity (Tukey HSD test: $p$ values between 0.00 and 0.01). The same test showed that the effect on the B4 interburst interval of 60 CGC spikes/min was significantly different from those at 12 spikes/min (Tukey HSD test: $p=0.002$ ) and 24 spikes/min (Tukey HSD test: $p=0.04)$. Furthermore, the increases in B4 burst frequency caused by 12 CGC spikes/min and 60 CGC spikes/min were significantly different (Tukey HSD test: $p=$ 0.006).

The data suggested that direct effects of CGC activity on the B4 motoneuron bursting frequency could be part of the modulatory mechanism involved in feeding motor program control reported to occur in the intact feeding system (Yeoman et al., 1994a,b).

\section{Intrinsic PIR properties in retraction phase motoneurons are enhanced by 5-HT and firing of the CGCs}

Spike activity in B4, B8, and B4CL motoneurons during fictive feeding is thought to be caused by PIR resulting from the N2 CPG inhibitory synaptic inputs (Benjamin and Rose, 1979; Fig. $1 C)$. Here we show that the PIR mechanism in these neurons is a target for modulation by 5 -HT.

The presence of a basic PIR mechanism in the B4, B8, and B4CL motoneurons could be demonstrated in cell culture by injecting hyperpolarizing current pulses and examining their rebound responses (Fig. 5A). However, the levels of hyperpolarization that were usually required to elicit a PIR response exceeded the amplitude of the normal physiological inhibitory synaptic inputs encountered in the intact nervous system (Benjamin and Rose, 1979). The size of the PIR response in all three types of motoneurons could be considerably enhanced by the application of 5-HT to the isolated cells (Fig. 5B). Before 5-HT application, the negative current pulses either failed to evoke, or only evoked small rebound depolarizations. These were considerably increased after brief focal applications of 5-HT (1 sec, $0.1 \mathrm{~mm})$. In the examples shown in Figure $5 B$, the effects were particularly striking in the B8 cell in which enhanced PIR effects were strong enough to evoke spikes $20 \mathrm{sec}$ after exogenous 5-HT application, and the PIR enhancement persisted for up to $80 \mathrm{sec}$.

Artificial depolarization of the membrane potential by positive current injection to the same value as reached during 5-HT application did not significantly affect the shape of responses to negative current pulses (Fig. $5 B$, right side traces; $n>4$ for all three cell types). This indicated that the modulation of the 
A
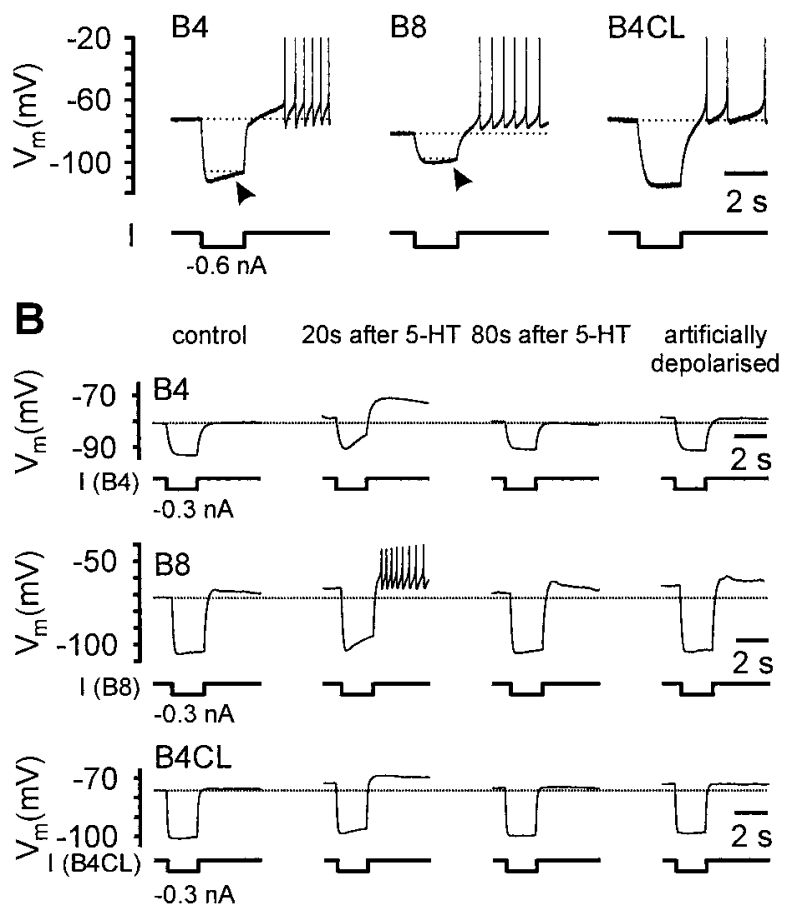

C
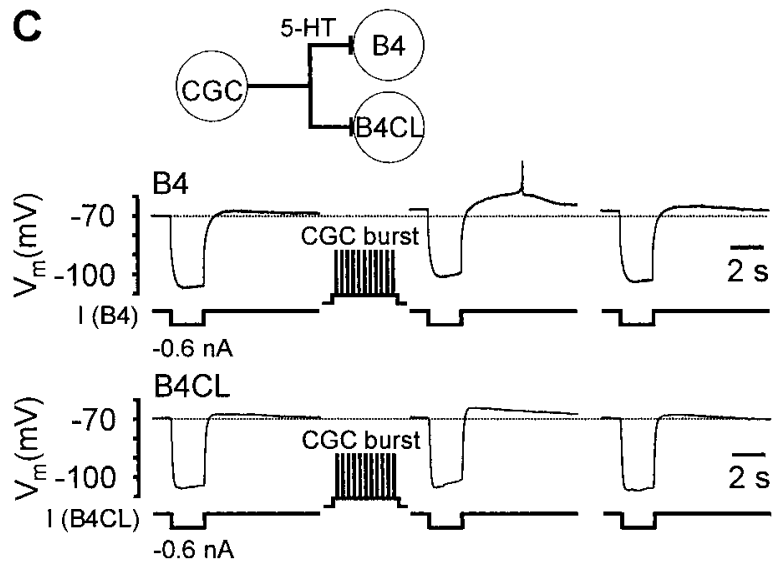

Figure 5. PIR properties of isolated B4, B8, and B4CL neurons in cell culture. $A$, Strong hyperpolarizing responses in $\mathrm{B} 4, \mathrm{~B} 8$, and $\mathrm{B} 4 \mathrm{CL}$ neurons elicited depolarizing overshoots after the end of the current pulses and evoked series of spikes in each of the three neurons. Note the gradual recovery of the membrane potential during the duration of the negative current pulse (arrows) that was particularly strong in the B4 neuron and somewhat weaker in the $\mathrm{B} 8$ and $\mathrm{B} 4 \mathrm{CL}$ neurons shown. $B$, The PIR properties in isolated B4, B8, and B4CL neurons were increased by brief applications of 5-HT. The records on the left represent controls before the application of 5-HT. Under these control conditions negative current pulses only evoked a weak PIR response in the B8 neuron and no consistent response in the B4 and B4CL neuron shown. Twenty seconds after the application of a brief 5-HT pulse $(0.1 \mathrm{~mm}, 1 \mathrm{sec})$, the same test pulses evoked considerably stronger PIR responses in all three neuron types and now triggered a series of spikes in the B8 neuron. Eighty seconds after 5-HT application the responses to the negative test pulses had returned to control levels in the B4 and B4CL neuron, but were still slightly elevated in the B8 neuron. Subsequently, the individual neurons were artificially depolarized by injection of constant positive currents to membrane potential values comparable with those obtained during 5-HT depolarization. This artificial depolarization did not alter the response of these neurons to the negative test pulses when compared with the control records. $C$, Records from a CGC that had reformed chemical synapses with a pair of cocultured B4 and B4CL neurons. Before CGC stimulation, negative current pulses only evoked weak PIR responses in both the B4 endogenous PIR properties in these neurons was a direct effect of 5-HT and not caused indirectly by the 5-HT-induced depolarization.

We predicted that 5-HT release from CGCs should have the same effects on PIR in retraction phase motoneurons as focal application of 5-HT. This was examined for B4 and B4CL neurons by coculturing the cells with CGC neurons (CGC-B4, $n=6$; CGC-B4CL, $n=3$ ). A burst of CGC spikes applied just before PIR was evoked by injection of a negative current pulse enhanced the size of the PIR for both cell types (Fig. 5C), consistent with the release of 5-HT from the CGCs.

When a negative current pulse elicited a PIR depolarization after the end of the pulse, the voltage trajectory during the pulse showed an initial negative peak after which the membrane potential started to slowly depolarize despite the continued current injection (Figs. $5 A$, arrows, $6 A$ ). We refer to this depolarization as a "sag" potential (c.f. Arbas and Calabrese, 1987; Angstadt and Friesen, 1993a,b; Zhang and Harris-Warrick, 1994). The existence of this sag was suggestive of the presence of a hyperpolarization-activated inward current, $I_{\mathrm{h}}$, in retraction phase motoneurons. $I_{\mathrm{h}}$ currents are typically activated after hyperpolarization of the membrane beyond the resting potential and show a high sensitivity to blockage by external $\mathrm{Cs}^{+}$ions (Pape, 1996). We used this sensitivity to $\mathrm{Cs}^{+}$ions to obtain evidence that the activation of an $I_{\mathrm{h}}$ current underlies the PIR properties in retraction phase motoneurons. In the presence of $\mathrm{CsCl}$, we showed that the PIR depolarization elicited by constant negative current pulses in both B4 and B4CL neurons was reduced significantly to $15 \pm 7 \%$ (B4: $t$ test, $p<0.01 ; n=3$ ) and to $13 \pm 9 \%$ (B4CL: $t$ test; $p<0.02 ; n=3$ ) compared with normal saline (Fig. $6 A, B$ ). $\mathrm{CsCl}$ also abolished the sag potential response that occurred during negative current pulses resulting in a considerable increase in the peak amplitude of the hyperpolarizing membrane potential excursion (Fig. 6A). The $\mathrm{CsCl}$ block was reversed to $\sim 80 \%$ of the control value after washing with normal saline for $\sim 5 \mathrm{~min}$ (Fig. $6 \mathrm{~B}$ ). The $\mathrm{CsCl}$ block of the sag potential response and the PIR depolarization support the hypothesis that activation of an $I_{\mathrm{h}}$ current is responsible for both of these effects. However, without further characterization of the ionic currents in these neurons that would go beyond the scope of the current paper, we cannot exclude the possibility that some other $\mathrm{CsCl}$ sensitive ion currents may also be involved.

Under voltage-clamp conditions hyperpolarizing holding potential steps induced a slowly activating inward current in isolated B4 and B4CL neurons. The amplitude of the inward current was increased by 5-HT (Fig. 6C, arrows) and strongly reduced by application of $\mathrm{CsCl}$ (Fig. 6C). Before application of 5-HT, a $2 \mathrm{sec}$ square-wave step in holding potential from -80 to $-120 \mathrm{mV}$ evoked an inward current that gradually increased in amplitude. When the membrane potential was stepped back to $-80 \mathrm{mV}$, a weak slowly inactivating inward tail current was recorded. The application of 5-HT increased significantly the amplitude of this tail current by approximately doubling it in both B4 and B4CL neurons (B4: $t$ test control vs 5-HT, $p<0.05, n=4$; B4CL: $t$

$\leftarrow$

and B4CL neuron (left records). After a $10 \mathrm{sec}$ burst of CGC activity between test pulses, the same test pulses elicited considerably stronger PIR responses that triggered a single spike in the B4 neuron (middle records). Forty seconds later the membrane potential and PIR response in the B4 neuron were still slightly elevated, whereas they had returned to the control levels in the B4CL neuron. 
Figure 6. Block of PIR by $\mathrm{CsCl}$ and effects of 5- $\mathrm{HT}$ and $\mathrm{CsCl}$ on a hyperpolarization-activated inward current in $\mathrm{B} 4$ and $\mathrm{B} 4 \mathrm{CL}$ neurons. $A$, Current-clamp recordings from isolated $\mathrm{B} 4$ and B4CL neurons in cell culture showing a PIR depolarization in response to $2 \mathrm{sec}$ negative current pulses. The PIR depolarization as well as the sag in membrane potential during the current pulse were completely abolished by the addition of $5 \mathrm{~mm}$ $\mathrm{CsCl}$ to the medium. The block of the sag resulted in an apparent increase in input resistance. The addition of $\mathrm{CsCl}(5 \mathrm{~mm})$ to the bath medium also caused a hyperpolarization of the membrane potential that was counteracted by injecting a constant positive current. The block was readily reversed after removal of $\mathrm{CsCl}$ from the bath. $B$, Summary of the data from three B4 and three B4CL neurons. The histogram shows the mean peak PIR amplitude (+ SEM) normalized to the peak PIR amplitude during the control period. $C$, TEVC current recordings from isolated $\mathrm{B} 4$ and B4CL neurons in cell culture. Under control conditions stepping the membrane potential for $2 \mathrm{sec}$ from the holding potential of -80 to $-120 \mathrm{mV}$ to mimic the effects of negative current pulses that were used to elicit PIR responses under currentclamp conditions caused an inward current that gradually increased in amplitude. The inward current gave rise to a weak slowly inactivating inward tail current when the holding potential was stepped

$A$
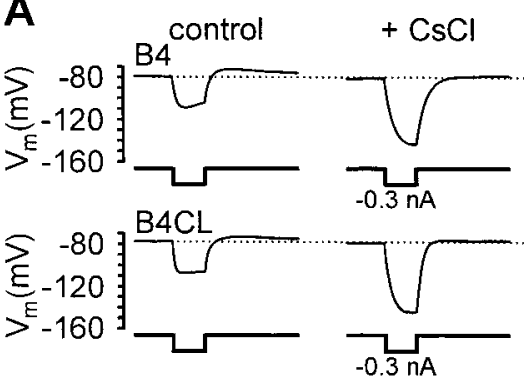

C.

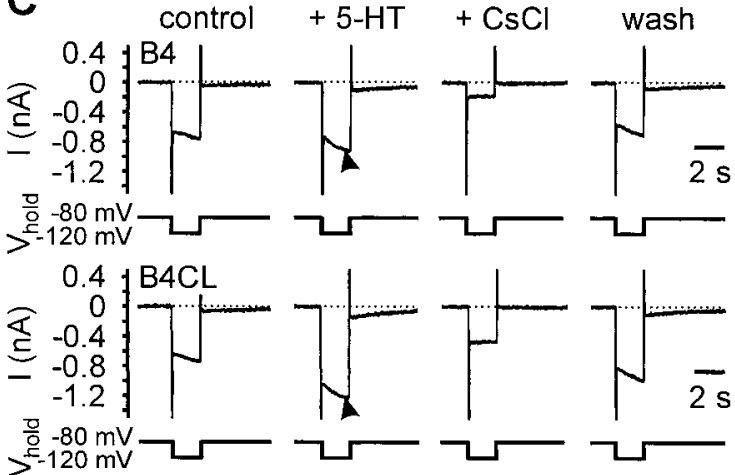

B

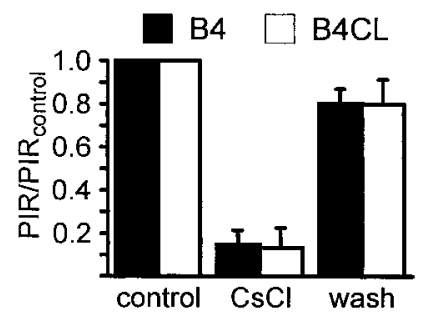

D

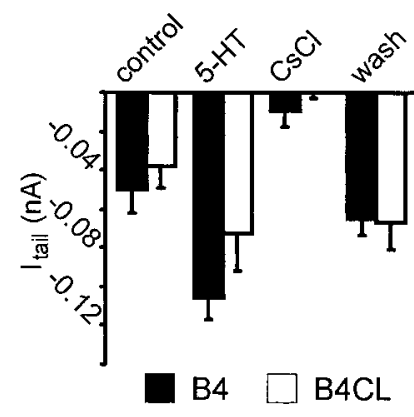

back to $-80 \mathrm{mV}$. The amplitude of the tail current in both B4 and B4CL neurons was significantly increased by the bath application of $0.1 \mathrm{~mm} 5$-HT (arrows). Subsequent exchange of the bath medium for NS containing $5 \mathrm{~mm} \mathrm{CsCl} \mathrm{completely} \mathrm{abolished} \mathrm{the} \mathrm{tail} \mathrm{current} \mathrm{and} \mathrm{significantly} \mathrm{reduced} \mathrm{the}$ current response during the potential step. Replacing the bath medium with NS readily reversed the effect of CsCl. $D$, Summary of the results for four B4 and five B4CL neurons. The data are presented as mean values plus SEM.

test $_{\text {control vs } 5 \text {-HT }}, p<0.05, n=5$; Fig. $6 D$ ). Subsequent exchange of the bath medium for a saline solution containing $5 \mathrm{~mm} \mathrm{CsCl}$ strongly reduced or completely abolished the 5-HT enhanced tail currents in B4 and B4CL neurons to values that were not significantly different from zero (one sample $t$ tests: B4: $p=0.36, n=$ 4; B4CL: $p=0.95, n=5$; Fig. $6 D$ ). Furthermore, this reduction was significant when compared with the 5-HT-enhanced tail currents (B4: $t$ test ${ }_{5-\mathrm{HT}}$ vs $\mathrm{CsCl}_{1}, p<0.01, n=4$; B4CL: $t$ test ${ }_{5-\mathrm{HT} \text { vs }}$ $\mathrm{CsCl}, p<0.05, n=5$ ). The $\mathrm{CsCl}$ block of the tail current was reversible when the bath medium was exchanged for normal saline. Approximately 15 min into the wash the amplitudes of the tail currents in B4 and B4CL neurons were reduced compared with the situation during 5-HT application but still enhanced compared with the original control values. The reduction in the tail current amplitude after wash compared with the presence of 5-HT was only just significant for B4 neurons ( $t$ test 5 -HT vs wash: $p=0.05, n=4)$ and not significant for B4CL neurons ( $t$ test control vs wash: $p=0.49, n=5)$. In contrast, the elevation of the tail currents after wash compared with control values was significant for the B4CL neurons ( $t$ test control vs wash: $p<0.01, n=5$ ), but not for the B4 neurons ( $t$ test control vs wash: $p=0.12, n=4)$. One possible explanation for the elevated washout response is that there may have been long-lasting residual effects from the 5-HT application, which became effective again after $\mathrm{CsCl}$ application had ceased. This is consistent with the long duration of the modulatory 5-HT effects on the membrane potential and endogenous bursting properties (Fig. 2).

\section{5-HT and CGCs effects on motoneurons in the intact nervous system}

Experiments on the retraction phase motoneurons in cell culture allowed the effects of 5-HT and CGC activity to be examined directly. However, it was important to show similar effects on motoneuron cellular properties in the buccal ganglion circuit to obtain evidence that these effects could contribute to pattern generation in the intact system and were not artifacts resulting from the culture system.

The effect of CGC firing frequency on the B4 PIR property was studied by recording simultaneously from both neurons in the isolated ganglia, while manipulating steady CGC activity $(n=3)$. When spontaneous CGC activity had ceased, which usually occurred in the isolated nervous system 60-90 min after initial impalement, repolarization at the end of the negative current pulses failed to reveal any consistent PIR depolarization in B4 neurons (Fig. 7A). However, when the $\mathrm{CGC}$ firing was reactivated subsequently by current injection, PIR depolarization could be elicited immediately in B4 neurons, and this progressively increased in amplitude with increasing $\mathrm{CGC}$ firing rates leading to the generation of $\mathrm{B} 4$ activity $(0-60$ spikes/min; Fig. $7 A, B)$. The strongest changes in PIR amplitude occurred at CGC firing rates between zero and 12 spikes/min (Fig. 7B). These rates are within the normal CGC firing range recorded by fine wires in the intact snail during food-stimulated feeding (Yeoman et al., 1994a). An ANOVA showed that significant differences existed between the B4 PIR amplitude at the CGC firing rates tested (ANOVA: $\left.F_{(5,23)}=4.49, p<0.01\right)$. A post hoc Tukey HSD test for multiple comparison revealed significant differences between the PIR amplitude at $60 \mathrm{CGC}$ spikes/min compared with 0 spikes/min $(p<$ $0.05)$ or 6 spikes/min $(p<0.05)$.

The effect of CGC activity on the B4 PIR amplitude was mimicked by bath application of 5-HT to isolated buccal ganglia. For these experiments, synaptic connections between the CGC neurons and the feeding system were severed by cutting the cerebrobuccal connectives to remove as far as possible any effects resulting from CGC activity. Furthermore, the isolated buccal 
A
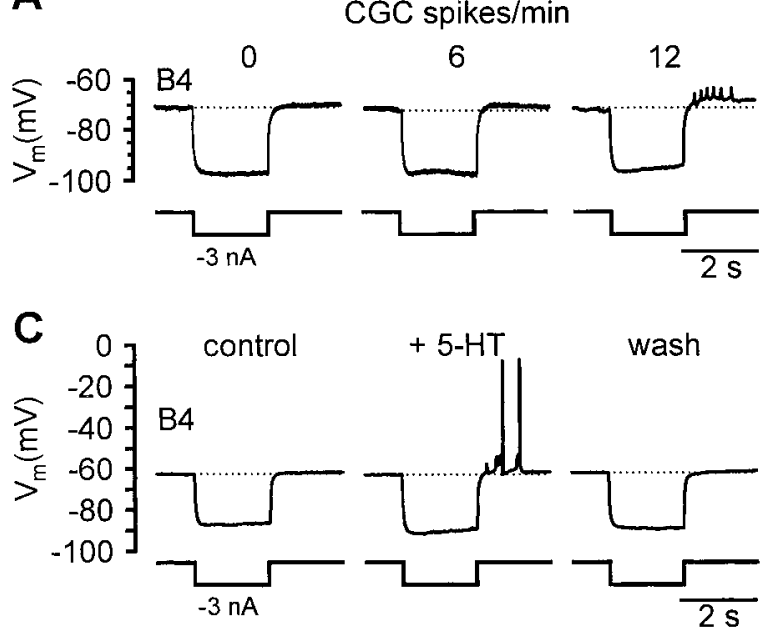

$E+d-T C$

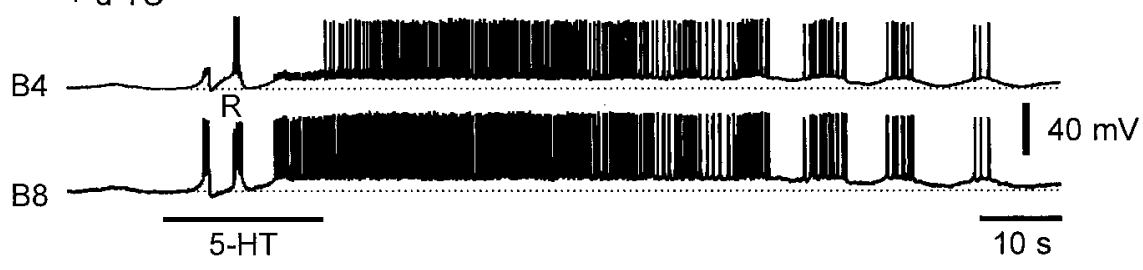

B

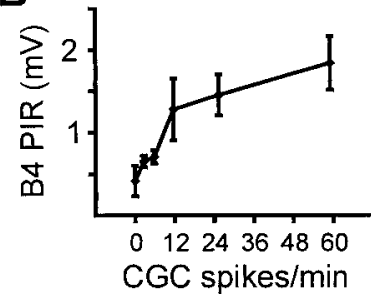

D

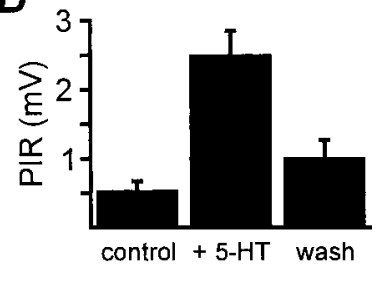

Figure 7. CGC and 5-HT effects on B4 neurons in the intact nervous system. $A$, Modulatory effect of CGC frequency on B4 PIR property. Sample records from a B4 neuron that was recorded simultaneously with a CGC neuron (data not shown). When the CGC was inactive, negative current pulses ( $2 \mathrm{sec},-3 \mathrm{nA})$ injected into the $\mathrm{B} 4$ neuron caused a square wave hyperpolarization of the membrane potential that returned rapidly to the resting potential after the end of the current injection, without the generation of a consistent PIR depolarization (left record). At 6 CGC spikes/min a weak PIR depolarization can be seen on the B4 record in response to the same negative current pulse (middle record). When the CGC frequency was raised to values of 12 spikes/min, the B4 PIR depolarization increased considerably in amplitude sufficient to trigger a burst of axonal spikes (right record). B, Mean peak B4 PIR amplitude (+ $\mathrm{SEM})$ recorded in three preparations in response to constant negative current pulses $(-3 \mathrm{nA}, 2 \mathrm{sec})$ at CGC frequencies ranging from $0-60$ spikes $/ \mathrm{min}$ (see Results for statistical analysis). $C$, Effect of 5-HT application on B4 PIR in isolated buccal ganglia. Record from a B4 neuron after buccal ganglia were separated from the rest of the nervous system by cutting the cerebrobuccal connectives. The isolated buccal ganglia were constantly perfused with d-TC $(0.1 \mathrm{~mm})$ to block cholinergic synapses and suppress fictive feeding activity. Under these conditions negative current pulses $(-3$ $\mathrm{nA}, 2 \mathrm{sec}$ ) failed to elicit a PIR depolarization (left record), comparable with the situation in records $A$ in the absence of CGC activity. After the bath application of 5-HT, identical current pulses caused a significant PIR depolarization that triggered the generation of action potentials (middle record). 5-HT also caused a direct depolarization of the membrane potential that was readjusted to control levels by the injection of a constant negative current. The enhancement of the B4 PIR property was reversed after washout of 5-HT for periods in excess of 10 min (right record). D, Mean peak PIR amplitude ( \pm SEM) recorded in six B4 neurons in isolated buccal ganglia in response to negative current pulses $(-3 \mathrm{nA}, 2 \mathrm{sec})$ in the absence and presence of 5 -HT $(0.1 \mathrm{~mm})$. E, Simultaneous records from a B4 and B8 neuron in the intact nervous system. The preparation, as in the previous records, was constantly perfused with d-TC (0.1 mM). Bath application of 5-HT $(0.1 \mathrm{mM}, 20 \mathrm{sec})$ caused a prolonged depolarization in both neurons that evoked strong activity first in the B8 and then also in the B4 neuron. After $\sim 60 \mathrm{sec} 5$-HT-induced activity in these neurons turned from tonic to rhythmic bursting. At the start of the 5-HT application, 5-HT appeared to trigger a single isolated rasp phase CPG input (marked $R$ ) but without activating a sequence of rhythmic inputs as would be expected during CPG-driven fictive feeding.

ganglia were bathed in tubocurarine to eliminate spontaneous and 5-HT-induced fictive feeding activity by blocking cholinergic synaptic connections from protraction phase interneurons such as N1Ms and SO (Elliott and Kemenes, 1992; Yeoman et al., 1996). Under these conditions, the injection of negative current pulses $(-3 \mathrm{nA}, 2 \mathrm{sec}$ at $30 \mathrm{sec}$ intervals) only elicited a weak PIR response in all $\mathrm{B} 4$ neurons tested $(0.52 \pm 0.16 \mathrm{mV}, n=6$; Fig. $7 C, D)$ comparable with the previous results, observed in the absence of CGC activity (compare Fig. 7, $B, D$ ). Bath application of 5-HT ( $0.1 \mathrm{~mm}, 45 \mathrm{sec})$ increased the PIR amplitude fivefold to $2.49 \pm 0.36 \mathrm{mV}\left(n=6 ; t\right.$ test $_{\text {control vs } 5 \text {-HT }}, p=0.001$; Fig. $\left.7 D\right)$, which was sufficient to trigger axonal and soma spikes in B4 neurons (Fig. 7C). The direct depolarization of the B4 membrane potential by 5 -HT was prevented by the injection of a constant negative current. Thus, the membrane potential was kept close to the value before 5-HT application. The enhancement of the PIR amplitude reversed gradually after removal of 5-HT from the bath, but even after 5 min of washing with normal saline the mean PIR amplitude was still almost double the control value $(0.98 \pm$ $0.28 \mathrm{mV}, n=6 ; t$ test $_{\text {control vs wash, }} p=0.11$; Fig. $\left.7 D\right)$. It was impossible to directly test the effects of manipulating CGC firing on "endogenous" rhythmic burst activity in B4/B8 motoneurons, because this always generates indirect synaptic inputs to the motoneurons via CGC effects on CPG interneurons (Yeoman et al., 1994a). Therefore experiments concentrated on examining the effects of 5-HT on the bursting properties of the motoneu- rons. As in the previous pharmacological experiments, the buccal ganglia were isolated from the rest of the nervous system by cutting the cerebrobuccal connectives and the preparation bathed in tubocurarine to reduce spontaneous synaptic inputs. Under these conditions, 5-HT $(0.1 \mathrm{~mm}, 20 \mathrm{sec})$ caused a prolonged depolarization in co-recorded B4 and B8 neurons $(n>6$; Fig. $7 E$ ). The depolarization outlasted the 5-HT application and triggered sustained activity in both B4 and B8 neurons that turned into coordinated bursting activity after $\sim 1 \mathrm{~min}$. Although there were occasional instances of N2 CPG inputs (usually one or two at the start of the 5-HT application, one labeled R in Fig. 7E), there was no evidence for a rhythmic synaptic input that could account for the bursting activity pattern observed in the B4/B8 pair. We therefore conclude that 5-HT was capable of activating endogenous bursting in the B4/B8 motoneurons.

\section{DISCUSSION}

We have shown for the first time that the CGCs and their main transmitter, 5-HT, modulate the intrinsic properties of three types of retraction phase motoneurons in the molluscan feeding system. These B4/B8, swallow phase and B4CL, rasp phase motoneurons are part of the feeding $\mathrm{CPG}$ and influence rhythmic motor activity via their electrotonic coupling to CPG interneurons (Staras et al., 1998). All three cell types contribute significantly to the maintenance and phase setting of the feeding rhythm, and so the effects of the serotonergic modulation on their 
activity are significant in understanding the role of external modulation in the whole feeding circuit. Previous work has emphasized the behavioral (Yeoman et al., 1994a) and circuit level control of the feeding system by the CGC/5-HT (Yeoman et al., 1994b, 1996) and had shown that 5-HT mediated the CGCs synaptic effects on B4 motoneurons using a variety of antagonists (Tuersley and McCrohan, 1989). Here we show that the cellular properties of the motoneurons are influenced by 5-HT modulation, allowing us to link together the cellular and systems levels of analysis to produce a more complete understanding of feeding motor pattern generation.

\section{Modulation of PIR}

Firing in B4/B8/B4CL neurons during fictive feeding, examined in previous studies in the intact ganglion, was mainly caused by PIR (Benjamin and Rose, 1979). PIR was triggered by periodic inhibitory synaptic inputs from the N2 CPG interneurons (Fig. $1 B)$. However, recordings from isolated motoneurons in culture showed that PIR effects were very weak unless either 5-HT was present or spike activity from cocultured CGCs was evoked. This suggested that 5-HT modulation via release from the CGCs was necessary for the normal activation of the motoneurons by PIR. Two types of experiments, manipulating CGC spike activity and applying 5-HT, confirmed this in the intact ganglion. In the absence of CGC activity (zero firing rate), PIR was very small or absent. Consistent PIR was observed only when the CGCs fired in their usual tonic manner. There was a progressive increase in the amplitude of the PIR-induced depolarization with increases in CGC firing rates, and this was particularly obvious in the $0-12$ spike firing range, corresponding to the rates of firing in the CGCs that are required for food-induced feeding in the intact snail (fine wire recordings; Yeoman et al., 1994a). The modulatory effects of the CGCs on the intact Lymnaea feeding system are caused by its transmitter, 5-HT (pharmacological experiments in Yeoman et al., 1994a), so it was significant that application of 5-HT increased the strength of PIR in the intact ganglion. This consistently led to firing in response to a hyperpolarizing pulse (Fig. 7C).

Enhancement of PIR properties by 5 -HT has also been described in CPG systems in a variety of invertebrates and vertebrates (Angstadt and Friesen, 1993a,b; Gasparini and DiFrancesco, 1999). We have shown here that the modulation of a CsCl-sensitive, hyperpolarization-activated inward current appears to underlie the PIR enhancement. Similar currents (labeled $I_{\mathrm{h}}$ ) have been studied in a large number of systems, including the DG neurons of the crab (Kiehn and Harris-Warrick, 1992) and leech swim and heart motoneurons (Mangan et al., 1994; Olsen and Calabrese, 1996). As in these other systems, the Lymnaea current was slow to inactivate, contributing to the formation of a depolarizing overshoot after the end of the hyperpolarizing current pulse. A corresponding "tail current", recorded under voltage clamp, is the slowly inactivating component of the hyperpolarization-activated inward current responsible for the depolarization. The amplitude of this current and the corresponding tail current were increased by 5 -HT.

\section{Long-term modulation of membrane potential}

Changing membrane potential is another way to modulate neuronal activity in CPG neurons (Flamm and Harris-Warrick, 1986a,b), which occurred in all three types of Lymnaea motoneurons with the application of 5-HT. Previous experiments on the intact ganglion had shown that the CGCs spikes could generate
1:1 slow 3-4 sec duration EPSPs in the B4/B4CL cells (McCrohan and Benjamin, 1980) that were mediated by 5-HT (Tuersley and McCrohan, 1989). The present experiments showed that 5-HT could have much longer-term modulatory effects on neuronal activity that lasted for many minutes.

In isolated cells, a $1 \mathrm{~min}$ bath application of $0.1 \mathrm{~mm} 5-\mathrm{HT}$ induced spike activity lasting for $>10 \mathrm{~min}$. This could not be attributable to persistent 5-HT in the experimental chamber because the continuous perfusion of normal saline washed out drugs within a few seconds. Similarly, shorter duration focal application of 5-HT also produced long-lasting effects on the B4/B8 and B4CL neurons. It clearly implies that membrane conductances are being affected for long periods, presumably via G-protein-coupled receptor second messenger mechanisms. cAMP-dependent $\mathrm{Na}^{+}$currents exist in Lymnaea B4 motoneurons and in the homologous Helisoma B19 neurons, where they are activated by 5-HT (McCrohan and Gillette, 1988; Price and Goldberg, 1993).

Similar long-term depolarizing effects occurred in the intact ganglion (Fig. $7 E$ ) and could provide an explanation for the ability of the CGCs to drive B4/B4CL neurons into rhythmic spike activity against a background of weak CPG synaptic input (Benjamin et al., 1981). Also a single burst of CGC activity can generate bursts of motoneuron activity that can far outlast the period of immediate CGC spike activity (Benjamin and Elliott, 1989).

\section{Conditional bursting}

The bursting activity of pattern generator neurons is often dependent on the presence of neuromodulators, and these "conditional bursters" are commonly found in CPG circuits (HarrisWarrick and Marder, 1991). Application of 5-HT to the B4/B8 neurons, both in culture and the intact ganglion, showed that Lymnaea motoneurons have a conditional bursting property that coincided with the 5-HT-induced depolarization. Simply depolarizing quiescent B4 or B8 motoneurons by current injection was not sufficient to produce bursting, so more complex conductance changes must be involved. When cocultured B4/B8 neurons reformed their normal electrotonic junction in culture, both cells burst in synchrony. This is the first clear demonstration of conditional bursting properties in the Lymnaea feeding circuit and it presumably contributes to B4/B8 bursting that is initiated by PIR. The more numerous B4CL neurons do not show bursting in response to 5-HT, and their responses are restricted to changes in membrane potential.

\section{Role of extrinsic modulation in the feeding network: a} contribution to the systems level analysis

We believe that the ability of 5-HT/CGC activity to modulate the intrinsic properties of the B4/B8/B4CL neurons in culture and the intact circuit has functional implications for the intact feeding circuit in both gating and frequency control.

\section{Gating control}

A simple mechanism for gating has been postulated in which the CGCs provide a background of excitation to the feeding network, which lowers the threshold for activation of the feeding CPG by food (Yeoman et al., 1996). CGC synaptic effects on feeding interneurons are indeed mainly excitatory, and although weak have significant effects on the spiking activity of individual cells (McCrohan and Benjamin, 1980; Yeoman et al., 1996). It now appears that the activity of feeding motoneurons is also significant in gating of the $\mathrm{CPG}$ because removal of motoneuron 
activity reduces or completely prevents CPG activation in SOdriven fictive feeding patterns (Staras et al., 1998). This indicates that any modulatory effects that increase the level of motoneuron firing will contribute to the gating function of the CGCs. The CGCs were previously thought to make a minor contribution to the activation of B4/B8/B4CL cells by providing a weak synaptic depolarization (McCrohan and Benjamin, 1980). Here we show that CGCs and their main transmitter, 5-HT, have much more significant excitatory effects than previously thought via effects on the intrinsic properties of the motoneurons including long-lasting depolarization, conditional bursting, and enhancement of PIR (see above). All three modulatory effects increase the probability of activity in the retraction phase motoneurons and therefore are likely to contribute to the gating function of the CGCs. The long duration of some of these modulatory effects can explain why the CGCs have such long-lasting modulatory effects on gating in the intact circuit (Yeoman et al., 1994a).

\section{Frequency control}

Increasing the mean firing rate of the CGCs from 0 to 40 spikes/ $\mathrm{min}$ in the intact nervous system produced a linear increase in the frequency of the fictive feeding rhythm that was mainly attributable to a reduction in the duration of the N1/protraction phase of the feeding rhythm (Yeoman et al., 1996). This was thought to be caused mainly by the CGCs exciting the N1Ms by direct and indirect synaptic pathways, leading subsequently to the more rapid transition from the $\mathrm{N} 1$ (protraction) to the $\mathrm{N} 2$ (retraction) phase (Yeoman et al., 1996). Here we have shown that CGCs can modulate directly the frequency of B4 endogenous bursting activity (Fig. 4), which could contribute to feeding frequency modulation in the intact network. Increasing CGC firing rates in culture within their physiological range increased the frequency of B4 bursting by mainly decreasing their interburst interval, with lesser effects on burst duration. This was consistent with data in the intact circuit where the CGCs increased the fictive feeding frequency without affecting the duration of the retraction phases of the feeding cycle.

\section{Network assembly}

In more general terms, CGC/5-HT modulation of B4/B8 and $\mathrm{B} 4 \mathrm{CL}$ neurons appears to be essential in configuring the retraction phase components of the whole pattern-generating network, and its function in the feeding pattern generating system appears to go beyond the simple excitation of individual neurons and extends to the functional assembly of the whole system. This resembles the situation in other pattern-generating systems, in particular the stomatogastric system, where various modulatory neurons can assemble distinct networks that produce unique activity patterns (Meyrand et al., 1994; Blitz et al., 1999).

\section{REFERENCES}

Angstadt JD, Friesen WO (1993a) Modulation of swimming behaviour in the medicinal leech. I. Effects of serotonin on the electrical properties of swim-gating cell 204. J Comp Physiol [A] 172:223-234.

Angstadt JD, Friesen WO (1993b) Modulation of swimming behaviour in the medicinal leech. II. Ionic conductances underlying serotonergic modulation of swim-gating cell 204. J Comp Physiol [A] 172:235-248.

Arbas EA, Calabrese RL (1987) Ionic conductances underlying the activity of interneurons that control heartbeat in the medicinal leech. J Neurosci 7:3945-3952.

Benjamin PR, Elliott CJH (1989) Snail feeding oscillator: the central pattern generator and its control by modulatory interneurons. In: Neuronal and cellular oscillators (Jacklet, JW, ed), pp 173-214. New York: Marcel Dekker.

Benjamin PR, Rose RM (1979) Central generation of bursting in the feeding system of the snail, Lymnaea stagnalis. J Exp Biol 80:93-118.
Benjamin PR, McCrohan CR, Rose RM (1981) Higher order interneurons which initiate and modulate feeding in the pond snail, Lymnaea stagnalis. In: Invertebrate neurobiology: mechanisms of integration (Salanki J, ed), pp 171-200. Oxford: Pergamon.

Blitz DM, Christie AE, Coleman MJ, Norris BJ, Marder E, Nusbaum MP (1999) Different proctolin neurons elicit distinct motor patterns from a multifunctional neuronal network. J Neurosci 13:5449-5463.

Brierley MJ, Staras K, Benjamin PR (1997a) Behavioral function of glutamatergic interneurons in the feeding system of Lymnaea: plateauing properties and synaptic connections with motor neurons. J Neurophysiol 78:3386-3395.

Brierley MJ, Yeoman MS, Benjamin PR (1997b) Glutamatergic N2v cells are central pattern generator interneurons of the Lymnaea feeding system: new model for rhythm generation. J Neurophysiol 78:3396-3407.

Elliott CJH, Benjamin PR (1985) Interactions of pattern-generating interneurons controlling feeding in Lymnaea stagnalis. J Neurophysiol 54:1396-1411.

Elliott CJH, Kemenes G (1992) Cholinergic interneurons in the feeding system of the pond snail Lymnaea stagnalis, II. N1 interneurons make cholinergic synapses with feeding motoneurons. Philos Trans R Soc Lond B Biol Sci 336:167-180.

Flamm RE, Harris-Warrick RM (1986a) Aminergic modulation of lobster stomatogastric ganglion. I. Effects on motor pattern and activity of neurons within the pyloric circuit. J Neurophysiol 55:847-865.

Flamm RE, Harris-Warrick RM (1986b) Aminergic modulation of lobster stomatogastric ganglion. II. Target neurons of dopamine, octopamine, and serotonin within the pyloric circuit. J Neurophysiol $55: 866-881$

Gasparini S, DiFrancesco D (1999) Action of serotonin on the hyperpolarization-activated cation current (I-h) in rat CA1 hippocampal neurons. Eur J Neurosci 11:3093-3100.

Harris-Warrick (1988) Chemical modulation of central pattern generators. In: Neural control of rhythmic movements in vertebrates (Cohen AH, Rossignol S, Grillner S, eds), pp 285-331. New York: Wiley.

Harris-Warrick RM, Marder E (1991) Modulation of neuronal networks for behavior. Annu Rev Neurosci 14:39-57.

Katz PS, Frost WN (1996) Intrinsic neuromodulation: Altering neuronal circuits from within. Trends Neurosci 19:54-61.

Kemenes G, Elekes K, Hiripi L, Benjamin PR (1989) A comparison of four techniques for mapping the distribution of serotonin and serotonin-containing neurons in fixed and living ganglia of the snail, Lymnaea. J Neurocytol 18:193-208.

Kemenes G, Hiripi L, Benjamin PR (1990) Behavioural and biochemical changes in the feeding system of Lymnaea induced by the dopamine and serotonin neurotoxins 6-hydroxydopamine and 5,6-dihydroxytryptamine. Philos Trans R Soc Lond B Biol Sci 329:243-255.

Kiehn O, Harris-Warrick RM (1992) 5-HT modulation of hyperpolarization-activated inward current and calcium-dependent outward current in a crustacean motor neuron. J Neurophysiol 68:496-508.

Kupfermann I, Weiss KR (1981) The role of serotonin in arousal of feeding behavior of Aplysia. In: Serotonin neurotransmission and behavior (Jacobs BL Gelperin A, eds), pp 255-287. Cambridge, MA: MIT.

Mangan PS, Curran GA, Hurney CA, Friesen WO (1994) Modulation of swimming behaviour in the medicinal leech. III. Control of cellular properties in motor neurons by serotonin. J Comp Physiol [A] 175:709-722.

McCrohan CR, Benjamin PR (1980) Synaptic relationships of the cerebral giant cells with motor neurones in the feeding system of Lymnaea stagnalis. J Exp Biol 85:169-186.

McCrohan CR, Gillette R (1988) Cyclic AMP-stimulated sodium current in identified feeding neurons of Lymnaea stagnalis. Brain Res 438:115-123.

Meyrand P, Simmers J, Moulins M (1994) Dynamic construction of a neural network from multiple pattern generators in the lobster stomatogastric nervous system. J Neurosci 14:630-644.

Olsen OH, Calabrese RL (1996) Activation of intrinsic and synaptic currents in leech heart interneurons by realistic waveforms. J Neurosci 16:4958-4970.

Orlovsky GN, Deliagina TG, Grillner S (1999) Neuronal control of locomotion. Oxford: Oxford UP.

Pape HC (1996) Queer current and pacemaker: the hyperpolarizationactivated cation current in neurons. Annu Rev Physiol 58:299-327.

Pentreath VW, Berry MS, Osborne NN (1982) The serotonergic cerebral cells in gastropods. In: Biology of serotonergic transmission (Osborne NN, ed), pp 457-513. New York: Wiley.

Price CJ, Goldberg JI (1993) Serotonin activation of a cyclic AMPdependent sodium current in an identified neuron from Helisoma trivolis. J Neurosci 13:4979-4987.

Ridgway RL, Syed NI, Lukowiak K, Bulloch AG (1991) Nerve growth factor (NGF) induces sprouting of specific neurons of the snail Lymnaea stagnalis. J Neurobiol 22:377-390.

Rose RM, Benjamin PR (1979) The relationship of the central motor 
pattern to the feeding cycle of Lymnaea stagnalis. J Exp Biol 80: 137-163.

Rose RM, Benjamin PR (1981a) Interneuronal control of feeding in the pond snail Lymnaea stagnalis. I. Initiation of feeding cycles by a single buccal interneurone. J Exp Biol 92:187-201.

Rose RM, Benjamin PR (1981b) Interneuronal control of feeding in the pond snail Lymnaea stagnalis. II. The interneuronal mechanism generating feeding cycles. J Exp Biol 92:203-228.

Rosen SC, Weiss KR, Goldstein RS, Kupfermann I (1989) The role of a modulatory neuron in feeding and satiation in Aplysia-effects of lesioning of the serotonergic metacerebral cells. J Neurosci 9:1562-1578.

Staras K, Kemenes G, Benjamin PR (1998) Pattern-generating role for motoneurons in a rhythmically active neuronal network. J Neurosci 18:3669-3688.

Tuersley MD, McCrohan CR (1989) Post synaptic actions of serotonergic cerebral giant cells on buccal motor neurones in the snail Lymnaea stagnalis. Comp Biochem Physiol 92C:377-383.

Weiss KR, Kupfermann I (1976) Homology of the giant serotonergic neurons (metacerebral cells) in Aplysia and pulmonate mollusks. Brain Res 117:33-49.

Weiss KR, Cohen JL, Kupfermann I (1978) Modulatory control of buc- cal musculature by a serotonergic neuron (metacerebral cell) in Aplysia. J Neurophysiol 41:181-203.

Yeoman MS, Pieneman AW, Ferguson GP, Ter Maat V, Benjamin PR (1994a) Modulatory role for the serotonergic cerebral giant cells in the feeding system of the snail, Lymnaea. I. Fine wire recording in the intact animal and pharmacology. J Neurophysiol 72:1357-1371.

Yeoman MS, Kemenes G, Benjamin PR, Elliott CJH (1994b) Modulatory role for the serotonergic cerebral giant cells in the feeding system of the snail, Lymnaea. II. Photoinactivation. J Neurophysiol 72:1372-1382.

Yeoman MS, Vehovszky A, Kemenes G, Elliott CJH, Benjamin PR (1995) Novel interneuron having hybrid modulatory-central pattern generator properties in the feeding system of the snail, Lymnaea stagnalis. J Neurophysiol 73:112-124.

Yeoman MS, Brierley MJ, Benjamin PR (1996) Central pattern generator interneurons are targets for the modulatory serotonergic cerebral giant cells in the feeding system of Lymnaea. J Neurophysiol 72:1372-1382.

Zhang B, Harris-Warrick RM (1994) Multiple receptors mediate the modulatory effects of serotonergic neurons in a small neural network. $\mathrm{J}$ Exp Biol 190:55-77. 